\title{
ANÁLISIS DE PROBLEMAS DE LA UNIVERSIDAD: EL CASO DE LA UNIVERSIDAD NACIONAL MAYOR DE SAN MARCOS, UNIVERSIDAD NACIONAL DE INGENIERÍA Y UNIVERSIDAD NACIONAL AGRARIA LA MOLINA
}

\author{
UNIVERSITY PROBLEM ANALYSIS: THE CASE OF THE NATIONAL UNIVERSITY \\ OF SAN MARCOS, NATIONAL UNIVERSITY OF ENGINEERING AND NATIONAL \\ AGRARIAN UNIVERSITY
}

\author{
Leoncio Fernández Jeri ${ }^{1}$
}

\begin{abstract}
Resumen
La Universidad pública peruana enfrenta diversos problemas, los cuales son necesarios identificarlos y analizarlos. La presente investigación enfoca su estudio en 3 universidades públicas muy representativas como son la Universidad Nacional Mayor de San Marcos (UNMSM), Universidad Nacional de Ingeniería (UNI) y Universidad Nacional Agraria La Molina (UNALM). Los datos fueron obtenidos mediante encuestas con cuestionarios aplicados a las autoridades de las 3 universidades. Los problemas encontrados fueron: La Ley universitaria, necesidad de un mayor presupuesto, falta de un modelo de gestión y la mejora de la calidad; siendo el más importante la referida al mayor presupuesto que es requerido en la universidad. En el análisis comparativo usando la prueba estadística Chi-cuadrado se concluye que si hay relación entre los problemas y universidades. La universidad peruana representada por estas 3 universidades tienen similares problemas, siendo el más importante la necesidad de mayor presupuesto $(91.25 \%)$, seguido de mejora de la calidad $(85.83 \%)$.
\end{abstract}

Palabras clave: Calidad, presupuesto, universidad

\begin{abstract}
The peruvian public university faces several problems, which are necessary to identify and analyze. This research study focuses on 3 very representative public universities such as the Mayoir National University of San Marcos, National Engineering University and National Agrarian University La Molina. Data were collected using a questionnaire survey applied to the authorities of the 3 universities.

The problems encountered were: The University Act, need a bigger budget, lack of model management and quality improvement; the most important being referred to as budget is required in college. In the comparative analysis using the statistical test Chi-square is concluded that there is a relationship between problems and universities. The Peruvian university represented by these 3 universities have similar problems, the most important being the need for more budget $(91.25 \%)$, followed by quality improvement $(85.83 \%)$.
\end{abstract}

Keywords: quality, budget, university

\section{Introduccion}

La educación del hombre es la base de su formación como persona, individuo de la sociedad e insumo para contribuir en la producción de su país, del bienestar de la sociedad y principalmente de su familia. Esta educación es importante a partir de la asistencia de las personas a la escuela y luego su ingreso a la universidad o educación superior. Antes de la escuela y la universidad, se llevará a cabo la etapa de formación en valores en el entorno familiar y en los años iniciales de colegio. Es en la etapa de la educación superior donde la universidad tiene la función principal de generar conocimiento, desarrollar el aprendizaje de las personas, y vincular la teoría con la práctica; logrando conseguir una persona con mucho conocimiento, vinculado a la práctica y decidido a contribuir en la sociedad para hacerla más productiva, formando empresa, investigando, y llegándose a constituir en capital humano.

La universidad pública peruana sigue el modelo universitario establecido en el año 1918. Si hay una organización que estructuralmente permanece igual por más de un siglo, esa es la Universidad Peruana. En nuestro país sólo existen 2 tipos de universidad: la pública y la privada. Entonces para hacer frente a la actual sociedad (y mercado) le resulta altamente restrictivo responder a sus demandas por su carácter rígido y funcional. Se estima que muchas opiniones inclusive de expertos en el tema universitario, señalan que nuestra universidad peruana 
atraviesa una crisis explicado por varios problemas. $\mathrm{Y}$ es allí donde surge la necesidad y motivación para realizar la presente investigación; para encontrar qué problemas tiene la universidad; enfocándonos en 3 prestigiosas universidades públicas, como son: La Universidad Nacional Mayor de San Marcos (UNMSM), La Universidad Nacional de ingeniería (UNI) y la Universidad Nacional Agraria La Molina (UNALM).

La Universidad está dejando de cumplir el rol de la formación del capital humano que apoye al país a ser más competitivo. La universidad pública peruana a nivel de la región sudamericana, es la que menos ha aplicado importantes cambios en su institución o hecho avances en procesos como el de acreditación para responder al entorno actual. Asimismo, tiene la ley universitaria más antigua de la región, ya que data del año 1983, y se espera pronto tener una nueva Ley.

La Universidad pública peruana enfrenta diversos problemas, los cuales son necesarios identificarlos y analizarlos. La presente investigación enfoca su estudio en 3 universidades públicas muy representativas; las cuales podrían usar los resultados de la presente investigación para aplicar políticas más efectivas que mejoren la situación de su institución.

\section{Objetivo General}

Analizar los principales problemas en las universidades peruanas: Universidad Nacional Mayor de San Marcos (UNMSM), Universidad Nacional de Ingeniería (UNI) y Universidad Nacional Agraria La Molina (UNALM)

\section{Objetivos específicos}

- Describir los problemas de la universidad peruana.

- Identificar los principales problemas de la universidad peruana, para el caso de la UNMSM, UNI y UNALM

- Comparar los problemas identificados, entre las 3 universidades, la UNMSM, UNI y UNALM

\section{Materiales y métodos}

Diseño de la investigación

El análisis se hace principalmente mediante encuestas a autoridades o directivos de las 3 universidades (UNMSM, UNI y UNALM). Los resultados cualitativos de las encuestas son luego evaluados con técnicas estadísticas cuantitativas como la prueba Chi-cuadrado.

Fuentes primarias y secundarias

Fuente primaria: Resultados de las encuestas aplicadas a las autoridades: Rectores, o vicerrectores o decanos de las universidades públicas de San Marcos (UNMSM), Ingeniería (UNI) y Agraria (UNALM).

Fuentes secundarias: Publicaciones, artículos y resultados de entrevistas realizadas a expertos, así como a ex autoridades (rectores, vicerrectores) e investigadores especializados en el área de educación superior.
Tamaño de muestra y los sujetos de encuesta

El tamaño de muestra es "a criterio del investigador" ( $a$ juicio); lográndose una cobertura mínima por universidad igual al $34.7 \%$, y en el total (24 autoridades) se cubrió el $50 \%$.

La encuesta se aplicó a las autoridades universitarias. La condición de "autoridad" se definen en los artículos correspondientes (art. 76 del Estatuto UNMSM, y art. 15 del estatuto UNI); nombrándose para ello a los rectores, vicerrectores, y/o decanos.

En la siguiente tabla se expone el número de encuestados por universidad así como el total.

Tabla 1. Número de Autoridades a ser encuestadas

\begin{tabular}{cccc}
\hline $\begin{array}{c}\text { Universidad } \\
\text { Pública }\end{array}$ & $\begin{array}{c}\text { Total Autoridades } \\
\text { (Decanos, } \\
\text { vicerrectores y/o } \\
\text { Rector) }\end{array}$ & $\begin{array}{c}\text { Número de } \\
\text { encuestados }\end{array}$ & $\begin{array}{c}\text { Cobertura } \\
(\%)\end{array}$ \\
\hline UNMSM & $\begin{array}{c}\text { 20 decanos, } 2 \\
\text { vicerrectores, 1 } \\
\text { Rector. Total : 23 } \\
\text { autoridades } \\
\text { 11 decanos, 1er. y }\end{array}$ & 8 & $34.70 \%$ \\
UNI & $\begin{array}{c}\text { 2do. Vicerrectores, } \\
\text { 1 Rector. Total : 14 } \\
\text { autoridades }\end{array}$ & 8 & $57.10 \%$ \\
UNALM & $\begin{array}{c}\text { 8 decanos, 02 } \\
\text { vicerrectores, 1 } \\
\text { rector. Total : 11 } \\
\text { autoridades } \\
\text { Total }\end{array}$ & 8 & $72.70 \%$ \\
Autoridades & $\quad 48$ & 24 & $50 \%$ \\
\hline Fuente. Elaboración propia con datos del portal de cada universidad.
\end{tabular}

Análisis de la situación universitaria y diagnóstico de problemas en la universidad pública

El análisis consta de las siguientes etapas:

$1^{\circ}$. Diagnóstico de problemas de la universidad peruana hecho por ex autoridades, que son las siguientes:

\begin{tabular}{|c|c|c|}
\hline Nombre y apellidos & $\begin{array}{c}\text { Cargo } \\
\text { desempeñado }\end{array}$ & Universidad \\
\hline Antonio Mabres Torello & Rector & $\begin{array}{l}\text { UNIV. DE } \\
\text { PIURA }\end{array}$ \\
\hline Marcial Rubio Correa & $\begin{array}{c}\text { Vicerrector } \\
\text { Académico y actual } \\
\text { Rector }\end{array}$ & PUCP \\
\hline Javier Sota Nadal & Rector & UNI \\
\hline Manuel Burga & Rector & UNMSM \\
\hline $\begin{array}{l}\text { Francisco Delgado de la } \\
\text { Flor }\end{array}$ & $\begin{array}{c}\text { Vicerector } \\
\text { Administrativo y } \\
\text { Rector }\end{array}$ & UNALM \\
\hline Oswaldo Zegarra Rojas & Rector & UPCH \\
\hline
\end{tabular}


$2^{\circ}$ Diagnóstico de problemas de la universidad peruana hecho por expertos en el tema universitario.

$3^{\circ}$ Planteamientos de los problemas y definición del principal problema; obtenido a través de las encuestas a las autoridades universitarias. Para las alternativas del problema principal se usa como referencia lo encontrado en la primera parte con el diagnóstico realizado por ex autoridades y expertos $\left(1^{\circ} . y 2^{\circ}\right.$.). El análisis se complementa con una figura.

Los instrumentos usados para las encuestas son cuestionarios.

$5^{\circ}$ Prueba estadística Chi-cuadrado para evaluar la dependencia o independencia entre tipo de problema y tipo de universidad; con el fin de determinar si el problema es representativo y probar estadísticamente cuál es el principal problema. Se usa el paquete estadístico SPSS (Statistical Package for the Social Sciences).

\section{Prueba estadística de Chi-cuadrado:}

Esta prueba es aplicable para variables aleatorias discretas o continuas. No requiere que los datos a usar tengan una distribución normal. Es muy aplicado a las investigaciones sociales.

Entre sus opciones de uso están las siguientes pruebas: Homogeneidad, independencia o para bondad de ajuste.

Tenemos entonces dos valores de frecuencia para cada clase $\mathbf{i}$

oi: frecuencia observada (corresponde a los datos de la muestra)

ei: frecuencia esperada (corresponde al modelo propuesto)

La teoría estadística demuestra que la siguiente variable es apropiada para realizar una prueba de bondad de ajuste.

Definición: Estadístico para la prueba de bondad de ajuste Chi-cuadrado

$$
\chi^{2}=\sum_{i=1}^{k} \frac{\left(o_{i}-e_{i}\right)^{2}}{e_{i}}
$$

Es la distribución chi-cuadrado con $\mathrm{v}=\mathrm{k}-\mathrm{r}-1$ grados de libertad, donde $\mathrm{r}$ es la es la cantidad de parámetros de la distribución que deben estimar

Es una condición necesaria para aplicar esta prueba que $\forall$ ii, ei $\geq 5$

\section{Resultados y discusión}

El presente capítulo de resultados se inicia con una caracterización y diagnóstico de las universidades peruanas. Se identifica el principal problema de las 3 universidades, y se realizan comparaciones sobre los problemas identificados.

\section{Caracterización de las universidades peruanas: Públicas y privadas}

Según datos del II Censo Universitario (realizado entre marzo y julio del 2010 en todas las universidades públicas y privadas en el Perú), hay 100 Universidades con una población de 937,430 (docentes, alumnos y personal administrativo y de servicios). De los cuales aproximadamente son 783,000 estudiantes de pregrado y hay 59,000 docentes.

El II Censo nos muestra que tenemos 35 universidades públicas y 65 universidades privadas. El $40 \%$ de la población es de la universidad pública y el $60 \%$ pertenece a la Universidad privada; entre otras cifras que se muestran en el siguiente cuadro:

Las Universidades públicas más pobladas (nombres y población) son: Universidad Nacional Mayor de San Marcos (28,645), Universidad Nacional San Agustín de Arequipa (24,212), Universidad Nacional Federico Villarreal (23105), Universidad Nacional San Antonio Abad del Cusco (16,374). Entre las universidades privadas con mayor población están la Universidad Privada Alas Peruanas $(57,616)$ y la Universidad Privada Cesar Vallejo $(37,163)$. Los universitarios del Perú son principalmente provenientes de colegios estatales $(62.8 \%)$ y de colegios particulares, laicos o no escolarizados $(37.2 \%)$.

\section{Diagnóstico de la universidad pública por ex- autoridades universitarias}

En primer lugar se expone el diagnóstico realizado por ex directivos (Rectores) de las Universidades Piura, Católica (PUCP), UNI, UNMSM, UNALM, y UPCH. En el cuadro 2 se resume el diagnóstico de las ex autoridades. Estos fueron comparados con los resultados expuestos por investigadores $\mathrm{y} / \mathrm{o}$ especialistas en educación superior como Espinoza (2005), Ramírez (2005), Depaz (2006), Cieza (2006), entre otros; en la revisión de antecedentes.

ANTONIO MABRES, Ex Rector de la Universidad de Piura

Mabres (1994) en su documento sobre los problemas de la Universidad peruana, sostiene que se enfrenta una problemática que, aunque común, se manifiesta en distintos grados y bajo distintas formas. No obstante, de manera simplificada podría decirse que esos problemas son cinco: bajo nivel académico -lo cual está asociado a la dificultad de los egresados para obtener trabajo-, incertidumbre económica, escasa investigación de calidad, poquísimas relaciones de colaboración académica entre universidades, e inexistencia casi total de relación con el sector empresarial. Para el ex rector de la Universidad de Piura, el problema más grave, evidentemente, es el del bajo nivel académico.

MARCIAL RUBIO, ViceRector Académico de la PUCP -Rubio ${ }^{2}$, analiza_la transformación de la administración ${ }^{2}$ http://sisbib.unmsm.edu.pe/bibvirtualdata/libros/Educacion/univer_ 
universitaria, señalando algunas diferencias entre la universidad pública y privada, por ejemplo en que la primera es financiada por el Estado, y la segunda no. Dice que la Universidad no debe administrarse como una empresa, pero si enfocados en servicios de calidad $\mathrm{y}$ eficiencia.

\section{JAVIER SOTA NADAL, Ex Rector de la UNI}

- Sota $^{3}$, señala la necesidad de hacer gestión cuando expone : "Ensayemos pues, una norma real, una norma aterrizada, una que permita navegar, que permita poner un pie tras otro para avanzar, para construir desde abajo y no desde la nube, una norma que permita a la universidad ser gestionable y gobernable"

Sota, describe 04 problemas:

- La asamblea universitaria, el consejo universitario y el consejo de facultad son órganos, en realidad "gimnasios de retórica, caros simulacros de plaza pública, lugares en los que se plantan las banderas estamentales olvidándose de la universitaria, aquella que tiene que ver con sus funciones"

- El rector es un preocupado de la correlación de fuerzas entre los órganos, para poder gobernar; tiene que enfrentar los intereses individuales y de grupo; además de preocuparse por los votos.

- Un tercer problema es la ineficacia administrativa. En las pequeñas universidades se administra solo profesores y aulas y se está a expensas del financiamiento del Estado; y en universidades grandes cuando ya se tiene que hacer gestión económica y financiera, no se puede otorgar dicha función a cualquier profesor; pero coincide con Rubio, en que no se puede separar la gestión académica de la gestión administrativa

- Las universidades requieren al igual que los estados, estabilidad democrática, continuidad institucional y funcionamiento permanente.

\section{MANUEL BURGA, Ex Rector de la UNMSM}

- Burga et. al. (2005), escribe "El deterioro de la calidad, entre 1970 a la actualidad, 34 años, aproximadamente, podríamos decir se encuentra muy relacionado con el deterioro del presupuesto, y lógicamente de la remuneración del docente universitario. Esta, en el caso de la universidad pública peruana, ha llegado a situarse en los niveles mas bajos de América Latina y por eso consideramos que su recuperación será lenta en la próxima década".

\section{FRANCISCO DELGADO DE LA FLOR, Ex Rector de la UNALM}

-Delgado de la Flor (2001), manifiesta que se deben buscar direcciones de una gestión empresarial específica para cada universidad. "Es necesario establecer una peru/pdf/a02.pdf

idem gestión de calidad que responda a las nuevas exigencias" En realidad se trata de encontrar un nuevo modelo, y eso es un reto. El modelo que ayude a la universidad a cumplir sus fines; y a tener una gestión que responda a su entorno.

Tabla 2. Diagnóstico de la universidad pública, a cargo de ex autoridades

\begin{tabular}{|c|c|c|}
\hline $\begin{array}{l}\text { Nombre de la } \\
\text { ex autoridad }\end{array}$ & Universidad & $\begin{array}{c}\text { Diagnóstico (resumen) sobre } \\
\text { la universidad peruana }\end{array}$ \\
\hline $\begin{array}{l}\text { Antonio } \\
\text { Mabres }\end{array}$ & $\begin{array}{c}\text { Ex Rector } \\
\text { de la } \\
\text { Universidad } \\
\text { de Piura }\end{array}$ & $\begin{array}{c}\text { La problemática se } \\
\text { manifiesta en distintas } \\
\text { formas como: bajo } \\
\text { nivel académico, escasa } \\
\text { investigación de calidad, } \\
\text { poca colaboración entre } \\
\text { universidades, entre otros } \\
\text { Problemas identificados: } \\
\text { Nivel académico, } \\
\text { Investigación, Colaboración, } \\
\text { otros. }\end{array}$ \\
\hline Marcial Rubio & $\begin{array}{c}\text { Ex } \\
\text { ViceRector } \\
\text {-PUCP } \\
\text { (actual } \\
\text { Rector) }\end{array}$ & $\begin{array}{c}\text { La universidad no debe } \\
\text { administrarse como empresa } \\
\text { pero sí enfocada en calidad y } \\
\text { eficiencia. } \\
\text { Problemas identificados: } \\
\text { Modelo de gestión }\end{array}$ \\
\hline $\begin{array}{l}\text { Javier Sota } \\
\text { Nadal }\end{array}$ & $\begin{array}{l}\text { Ex Rector } \\
\text {-UNI }\end{array}$ & $\begin{array}{l}\text { Es necesario, hacer una } \\
\text { universidad gestionable y } \\
\text { gobernable. No se puede } \\
\text { separar la gestión académica } \\
\text { de la gestión administrativa. }\end{array}$ \\
\hline Manuel Burga & $\begin{array}{l}\text { Ex Rector- } \\
\text { UNMSM }\end{array}$ & $\begin{array}{l}\text { Problemas identificados: } \\
\text { Modelo de Gestión, } \\
\text { Hay deterioro de la calidad } \\
\text { y del presupuesto. Y es } \\
\text { necesario construir una } \\
\text { nueva universidad. } \\
\text { Problemas identificados } \\
\text { :Calidad, presupuesto }\end{array}$ \\
\hline $\begin{array}{l}\text { Francisco } \\
\text { Delgado de } \\
\text { la Flor }\end{array}$ & $\begin{array}{l}\text { Ex Rector } \\
\text {-UNALM }\end{array}$ & $\begin{array}{l}\text { Es necesario gestionar } \\
\text { la calidad. Para ello es } \\
\text { necesario encontrar un } \\
\text { nuevo modelo que ayude a } \\
\text { la universidad que cumpla } \\
\text { sus fines y responda al } \\
\text { entorno. } \\
\text { Problemas identificados: } \\
\text { Modelo de gestión, } \\
\text { calidad, competitividad }\end{array}$ \\
\hline $\begin{array}{l}\text { Oswaldo } \\
\text { Zegarra }\end{array}$ & $\begin{array}{l}\text { Ex Rector } \\
\text { UPCH }\end{array}$ & $\begin{array}{c}\text { Una nueva ley debe } \\
\text { contemplar un sistema } \\
\text { de elección "ponderado". } \\
\text { Problemas identificados: } \\
\text { Ley universitaria }\end{array}$ \\
\hline
\end{tabular}

OSWALDO ZEGARRA, ex Rector de la Universidad Particular Cayetano Heredia

-Zegarra es entrevista para la Revista del Instituto de 
derecho de la PUCP, IdehPucp (2012). En ella analiza y opina en el debate sobre la Ley 23733 que está pendiente de modificarse a través del Congreso desde el año 2005, y señala que: "El mecanismo que plantea para la elección de las autoridades es de carácter masivo; la elección universal dentro del claustro, con profesores y alumnos participando simultáneamente en un proceso electoral, supondría campañas un tanto demagógicas, con contenido político o con orientaciones no necesariamente adecuadas para la universidad; $y$ todo lo que signifique una elección universal con casi iguales criterios tanto de profesores y alumnos va a llevar a que los mejores profesores, y por tanto, con mejores posibilidades de ser rector".

\section{Los problemas de la universidad pública: El caso de la} UNMSM, UNI y UNALM

Para el diagnóstico, la descripción de la situación y definición del principal problema de la universidad pública, para el caso de las 3 universidades: Universidad Nacional Mayor de San Marcos (UNMSM), Universidad Nacional de Ingeniería (UNI) y Universidad Nacional
Agraria La Molina(UNALM); se propuso a las autoridades encuestadas señalar los problemas que existen en la Universidad pública, además de indicar ¿cuál sería el problema más importante, presentando como alternativas algunas ya manifestadas por ex autoridades?.

\section{El principal problema en las universidades: UNMSM, UNI y UNALM}

El principal problema para cada una de las 3 universidades En la siguiente tabla No. 3 se pueden observar los puntajes (\%) obtenidos en las encuestas:

-Las autoridades de la UNMSM asignaron mayor puntaje al problema: "falta de mayor

presupuesto" (90\%) seguido por la "mejorar la calidad"(83.75\%). Las autoridades de la UNI señalaron la necesidad de una "nueva ley Universitaria" como el problema más importante $(91.25 \%)$. Y todas las 8 autoridades encuestadas en la UNALM, asignaron el mayor peso (10 puntos cada uno) al problema "mayor presupuesto"; señalándolo entonces como el más importante.

Tabla 3. El principal problema de la universidad pública: UNMSM, UNI y UNALM

\begin{tabular}{cccccccc}
\hline $\begin{array}{c}\text { Problemas de las } \\
\text { universidades }\end{array}$ & $\begin{array}{c}\text { UNMSM } \\
\text { (puntaje) }\end{array}$ & $\begin{array}{c}\text { UNMSM } \\
(\%)\end{array}$ & UNI (puntaje) & UNI (\%) & $\begin{array}{c}\text { UNALM } \\
\text { (puntaje) }\end{array}$ & $\begin{array}{c}\text { UNALM } \\
(\%)\end{array}$ & $\begin{array}{c}\text { Total (\%) } \\
\text { Nueva Ley Universitaria }\end{array}$ \\
59 & $73.75 \%$ & 73 & $\mathbf{9 1 . 2 5 \%}$ & 51 & $63.75 \%$ & 76.25 \\
Mayor presupuesto & 72 & $\mathbf{9 0 \%}$ & 67 & $83.75 \%$ & 80 & $\mathbf{1 0 0 \%}$ & $\mathbf{9 1 . 2 5}$ \\
Modelo de Gestión & 65 & $81.25 \%$ & 59 & $73.75 \%$ & 69 & $86.25 \%$ & 80.42 \\
Mejorar calidad & 67 & $83.75 \%$ & 67 & $83.75 \%$ & 72 & $90 \%$ & 85.83 \\
\hline
\end{tabular}

Fuente. Elaboración propia con resultados de la encuesta a autoridades.

Considerando a las 3 universidades como representativas, en la fig. 1 podemos observar que el problema más importante de las 3 universidades es la necesidad de un "mayor presupuesto".

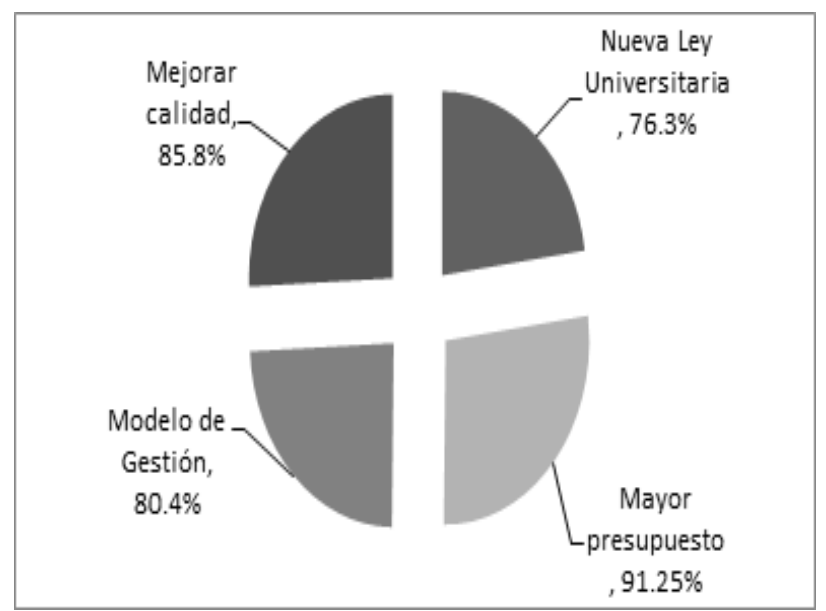

Figura 1. El problema más importante en la universidad pública, el caso de la UNMSM, UNI y UNALM Fuente: Elaboración propia y datos del cuadro 3.
Entre otros problemas que también fueron citados por las autoridades de las 3 universidades, están: La falta de investigación, infraestructura, innovación, capacitación, vinculo universidad-empresa.

¿Existe dependencia o independencia entre el tipo de Universidad y tipo de problema?

En el cuadro 4 se observa una relación en la definición de los problemas más importantes, por su mayor porcentaje. Así, la "necesidad de mayor presupuesto" es un principal problema en 2 de las 3 universidades (UNALM, UNMSM); y la necesidad de una "nueva ley universitaria" es el principal problema para el caso de la UNI. Y es relevante señalar que el tema del presupuesto se ubica como segundo problema más importante para esta universidad.

De la descripción y análisis anterior se podría afirmar que ¿la necesidad de un "mayor presupuesto" representa el problema principal de las 3 universidades? 
Prueba estadística de Chi-cuadrado $\left(\mathrm{X}^{2}\right)$ para probar la independencia entre tipo de universidad y tipo de problema.

Con el fin de usar la Prueba Chi-cuadrado para determinar si existe independencia entre el tipo de problema y universidad, se consideró como muestra, $\mathrm{n}=49$ casos, de un total de 96 (32 respuestas para cada una de las 3 universidades). En la siguiente tabla 4, observamos por ejemplo que 16 (autoridades) de las 49 respuestas (autoridades) coincidieron en definir la "falta de presupuesto" como el principal problema (asignación de 9 o 10 puntos para el problema); y que sólo en 7 casos se indicó como principal problema el "modelo de gestión".

Tabla 4. Cálculo para el análisis de independencia en la definición del principal Problema de la universidad.

\begin{tabular}{cccccc}
\hline & \multicolumn{6}{c}{$\begin{array}{c}\text { Modelo } \\
\text { Universidad }\end{array}$} & $\begin{array}{c}\text { Mejora } \\
\text { Ley }\end{array}$ & $\begin{array}{c}\text { Palta mayor } \\
\text { Presupuesto }\end{array}$ & $\begin{array}{c}\text { Tetal } \\
\text { Gestión }\end{array}$ & calidad & \\
\hline UNMSM & 5 & 4 & 2 & 5 & 16 \\
UNI & 7 & 4 & 2 & 3 & 16 \\
UNALM & 1 & 8 & 3 & 5 & 17 \\
Total & $\mathbf{1 3}$ & $\mathbf{1 6}$ & $\mathbf{7}$ & $\mathbf{1 3}$ & $\mathbf{4 9}$ \\
\hline
\end{tabular}

Fuente. Elaboración propia a partir de los resultados de las encuestas.

Prueba de hipótesis:

Ho: Existe independencia entre la universidad y el tipo de problema

H1: No existe independencia entre la universidad y el tipo de problema

Prueba Chi-cuadrado $\left(\mathrm{X}^{2}\right)$

Para un nivel de significación de 0.05, y con (4-1)(3-1) gl encontramos un $\mathrm{X}^{2} \mathrm{tab}=1.6354$, y el cálculo del valor estadístico de $\mathrm{X}^{2}$ es igual a 7.067. Los valores del desarrollo de la prueba se obtuvieron usando SPSS v. 15. Como $\mathrm{X}^{2}$ calc $=7.067>\mathrm{X}^{2}(0.95,6 \mathrm{gl})=1.6354 \mathrm{se}$ concluye que rechazamos la hipótesis de independencia y entonces existe relación entre el tipo de universidad y tipo de problema.

\section{Conclusiones}

La situación de la universidad pública peruana, en opinión de ex autoridades se resume en: La necesidad de poder gestionar la universidad, con los objetivos de responder al entorno, mejorar su nivel académico y su calidad.

La situación en las universidades públicas: UNMSM, UNI y UNALM; presenta como su problema más importante la necesidad de un mayor presupuesto. En particular, en dos de las tres universidades consideradas (UNMSM, UNALM) en la presente investigación encontramos que hace les falta principalmente más presupuesto; y en la tercera universidad (UNI) se evidencia la necesidad de una nueva ley universitaria como el problema principal (y la necesidad de presupuesto, su segundo problema más importante).

Porúltimo, aplicando la prueba estadística correspondiente (Chi cuadrado) se concluye que si existe relación entre el tipo de problema y universidad. La universidad peruana representada por estas 3 universidades tiene similares problemas, siendo el más importante la necesidad de mayor presupuesto.

\section{Literatura citada}

Alianza Estratégica (2002). “Acta de constitución de la Alianza estratégica”. Documento del convenio suscrito por las universidades UNMSM, UNI y UNALM. Lima, Perú.

Brunner (2008). "El processo de Bolonia en el horizonte latinoamericano: limites y posibilidades". Revista de educación 2008. pp119-145.

Burga et al. (2005) "Temas de reflexión en torno a la universidad peruana”. Conversatorio Por una nueva reforma universitaria, 10 de mayo del 2005. UNMSM, Perú

Burga Díaz, Manuel (2005). ¿Nueva Reforma Universitaria o Nuevo Modelo de Universidad? Revista Educación UNMSM, Año II, vol. II, No. 3 , 2005. Lima, Perú.

Cieza Mora, Jairo (2006) "Importancia del buen gobierno universitario". Boletín No. 11 de la comisión de coordinación de reforma universitaria (CCRU). UNMSM. Perú.

Consorcio de Universidades (2007). "Informe: La educación superior en Iberoamérica 2006 Perú". Consorcio de Universidades, Miraflores- Lima, Perú. 55 pgs.

Delgado de la Flor et al (2001) . "La Gestión Universitaria" publicado en

http://sisbib.unmsm.edu.pe/bibvirtualdata/libros/ Educacion/univer_peru/pdf/a02.pdf, revisado el 24 de enero del 2009.

Depaz, Zenón (2006). “Gestión universitaria y promoción de la equidad”. Educared Perú, Fundación telefónica, junio del 2006. Perú

De Vries (2004). “La Gestión de la universidad”. Revista mexicana de investigación educativa 9:22. pg. 575-584. Consejo mexicano de investigación educativa.

Espinoza Herrera, Nemesio (2005). "Vigencia de las Universidad Públicas del Perú". Propuestas para la reestructuración universitaria, 78 pgs. Perú

Fernández Jeri, Leoncio (2008). "Análisis de la gestión universitaria en la Universidad pública peruana”. Exposición de Caso en el VIII Coloquio Internacional sobre gestión universitaria en América del sur, Asunción, Paraguay. 
Ferrer y Pelekais (2004). "Tendencias gerenciales y la gestión universitaria". Revista de ciencias sociales, Vol. X, No. 1, enero-abril del 2004, pp.48-163. Universidad Rafael Belloso Chacin, URBE. Venezuela.

Fraile, Antonio (2006). "El sistema universitario europeo como modelo posible para la educación superior latinoamericana". Revista Electrónica de investigación educativa. Vol 8. No. 01. Universidad autónoma de baja California, México. 16 pgs.

IdehPucp (2012). "Modificaciones a la Ley Universitaria". Revista del Instituto de derechos humanos de la PUCP, edición No. 42; año 2011. Lima-Perú

INEI (2011). "II Censo nacional universitario 2010: Principales resultados". Instituto Nacional de Estadística y Asamblea Nacional de Rectores, 456 pág. Lima- Perú.

Mabres, A. (1994). "Problemas y perspectivas de las universidades peruanas". Revista No. 12, octubre 1994; del grupo de análisis para el desarrollo GRADE. Perú.

Ministerio de Educación (2006). "La Universidad en el Perú", Razones para una reforma Universitaria. Dirección de Coordinación universitaria, Lima Perú, 198 pgs.

Nava, Hugo (2006). "Sobre la Ley del SINEACE". Comisión de coordinación de Reforma Universitaria (CCRU). Boletín No. 16- UNMSM. Lima. 6 páginas. 